\section{Rivaroxaban para el tratamiento de la trombocitopenia inducida por heparina. Caso clínico}

\author{
GUSTAVO RIVERA O. ${ }^{1, a}$, OSCAR CORSI S., ${ }^{2, b}$, ANDRÉS PAVLOVIC J., \\ ANDRÉS AIZMAN S. ${ }^{2}$, PABLO VARAS A. ${ }^{2,4}$
}

\section{Treatment of heparin-induced thrombocytopenia with rivaroxaban. Case report}

Heparin-induced thrombocytopenia (HIT) is immune-mediated. It occurs more frequently with unfractionated heparin (UFH) than with low molecular weight heparins (LMWH). It is associated with thromboembolic rather than hemorrhagic events, as opposed to thrombocytopenia of other etiologies. The key in therapy is the cessation of heparin and the start of another anticoagulant. We report a 58 years old female with HIT secondary to the use of Enoxaparin who was successfully managed with Rivaroxaban. Our goal is to report a novel therapy and provide the evidence that supports its use.

(Rev Med Chile 2017; 145: 1213-1217)

Key words: Abnormalities, Drug-Induced; Heparin; Rivaroxaban; Thrombocytopenia; Thrombosis.
'Escuela de Medicina, Pontificia Universidad Católica de Chile, Santiago, Chile.

2Departamento de Medicina Interna, Escuela de Medicina, Pontificia Universidad Católica de Chile, Santiago, Chile.

${ }^{3}$ Programa de Geriatría, Escuela de Medicina, Pontificia Universidad Católica de Chile,

Santiago, Chile.

${ }^{4}$ Servicio de Medicina, Complejo Asistencial Dr. Sótero del Río,

Puente Alto, Chile.

anterno de medicina.

${ }^{\text {b}}$ Residente.

Recibido el 13 de junio de 2017, aceptado el 29 de agosto de 2017.

Correspondencia a:

Pablo Varas Arancibia

Diagonal Paraguay 362, Oficina

512, Departamento de Medicina Interna. Escuela de Medicina, Pontificia Universidad Católica de Chile, Santiago. pnvaras@uc.cl
S e presenta el caso de una paciente de sexo femenino de 58 años con antecedentes de artritis reumatoide, hipotiroidismo, hipertensión arterial, diabetes mellitus tipo 2 no insulino-requirente y gonoartrosis derecha.

Se hospitaliza de forma electiva para artroplastía total de rodilla derecha, sin incidentes perioperatorios. Recibe profilaxis con enoxaparina $40 \mathrm{mg} / \mathrm{día}$ subcutáneo a continuar al alta. Al sexto día postoperatorio presenta aumento de volumen doloroso de la extremidad inferior derecha de instalación brusca, asociado a calor y eritema. Consulta al servicio de urgencia del Hospital Dr. Sótero del Río al $17^{\circ}$ día postoperatorio por progresión del cuadro. Ingresa en buenas condiciones generales, afebril, sin compromiso hemodinámico ni ventilatorio. Reingresa con hemoglobina de 11,1 g/dL; leucocitos $13.930 / \mathrm{mm}^{3}$; plaquetas $52.000 / \mathrm{mm}^{3}$, creatinina 1,39 $\mathrm{mg} / \mathrm{dL} ; \mathrm{BUN} 19 \mathrm{mg} / \mathrm{dL}$, PCR $52 \mathrm{mg} / \mathrm{L}(\mathrm{VN}<5)$, glucosa 96 mg/dL; INR 1,23, TTPa 31,2 seg. Traumatología descarta artritis séptica y solicita ecografía doppler que confirma trombosis venosa profunda de vena femoral común y poplítea derechas. Se hospitaliza en servicio de traumatología ajustando enoxaparina a dosis terapéutica $(1 \mathrm{mg} / \mathrm{kg} / 12 \mathrm{~h})$. En controles seriados de hemograma evoluciona con disminución de plaquetas hasta $20.000 / \mathrm{mm}^{3}$ ( $28^{\circ}$ día postoperatorio, $10^{\circ}$ día de Enoxaparina en dosis terapéutica). Evaluado por medicina interna, se sospecha HIT con score $4 \mathrm{~T}>6$ puntos. Se traslada a servicio de medicina y se define suspensión inmediata de enoxaparina e inicio de rivaroxaban. Se descartan causas de pseudotrombocitopenia y microangiopatías. Se solicitan anticuerpos anti heparina-PF4 que resultan positivos. Evoluciona favorablemente, con disminución del empastamiento y dolor, sin otros eventos trombóticos. $\mathrm{Al}$ $7^{\circ}$ día de rivaroxaban presenta recuperación del recuento plaquetario $>150.000 / \mathrm{mm}^{3}$. Se decide alta al $18^{\circ}$ día con hemoglobina de $9,7 \mathrm{~g} / \mathrm{dL}$, plaquetas $273.000 / \mathrm{mm}^{3}$ y función renal conservada. Se decide completar 3 meses de terapia con rivaroxaban. 


\section{Discusión}

La trombocitopenia inducida por heparina (Heparin-induced thrombocytopenia: "HIT") se presenta típicamente con caída del recuento plaquetario $>50 \%$ del basal, alcanzando un nadir entre 40.000 y $80.000 / \mathrm{mm}^{3} .1$. El cuadro clásico se desencadena 5 a 10 días posterior al inicio de heparina, pudiendo aparecer antes de los 5 días en casos de exposición reciente a heparínicos ${ }^{2,3}$.

La fisiopatología de HIT se basa en la activación y agregación plaquetaria mediada por complejos inmunes IgG-PF4/heparinoide. El factor plaquetario 4 (PF4) es una proteína con carga positiva almacenada en los gránulos alfa de las plaquetas que se libera cuando éstas son activadas (cirugías, trauma, sepsis). Normalmente se une a glicosaminoglicanos cargados negativamente en células endoteliales contiguas. Dado la mayor afinidad del PF4 por la heparina, esta desplaza el PF4 liberándolo de la vasculatura y sitios de unión celular a la circulación, permitiendo así la formación de complejos inmunogénicos PF4/heparina. Estos complejos son reconocidos por el sistema inmune (Linfocitos B), generando IgG anti PF4/hepari$\mathrm{na}^{1,2,4,5}$. Estos anticuerpos se unen a receptores plaquetarios produciendo agregación y liberación de mediadores procoagulantes (trombina). Así se explica el nexo de ciertas condiciones de riesgo para HIT, la trombocitopenia por consumo y la preponderancia de los eventos trombóticos por sobre eventos hemorrágicos.
Algunos de los factores de riesgo más importantes para el desarrollo de HIT son el sexo femenino y el uso de HNF (10 veces más riesgo que HBPM $)^{1,2,6}$. Asimismo, el riesgo de pacientes postoperados de cirugía cardíaca y traumatológica es mayor que en otros pacientes. Se describe una incidencia de 1 a $5 \%$ para uso de HNF y 0,1 a $1 \%$ para uso de $\mathrm{HBPM}^{7,8,9}$.

Los pacientes con HIT tienen 20 a 40 veces más riesgo de desarrollar trombosis que la población general $^{2,3}$. Se reportan complicaciones tromboembólicas en $50 \%$ de los casos confirmados, siendo más frecuentes la trombosis venosa profunda y el tromboembolismo pulmonar. El desarrollo de trombosis suele aparecer después de la caída del recuento plaquetario, pero puede ocurrir antes ${ }^{1}$. Se describe que la tasa de trombosis previa al inicio del tratamiento alcanza el $5 \%$ por día ${ }^{10}$.

Otras manifestaciones clínicas son: necrosis cutánea en los sitios de inyección de heparina, reacción anafilactoídea, fiebre, síndrome de distrés respiratorio, entre otras ${ }^{11}$.

Ante la sospecha de HIT, una de las herramientas más usadas para orientar el estudio es el "4T SCORE" (Thrombocitopenia, Timing, Thrombosis, $o$ Ther $)^{2,12}$. Este divide a los pacientes en 3 categorías de riesgo según variables clínicas, de laboratorio y sospecha de causas alternativas (Tabla 1). Así se define el riesgo en: bajo (score 0-3), moderado (4-5) y alto (6-8) $)^{1,12}$. Una revisión sistemática estimó un VPN de $99,8 \%$ para score bajo y VPP de $14 \%$ y $64 \%$ para scores moderado y

Tabla 1. 4T SCORE para la clasificación de riesgo de HIT

\begin{tabular}{|c|c|c|c|}
\hline \multirow[t]{2}{*}{ Criterio } & \multicolumn{3}{|c|}{ Puntaje asignado } \\
\hline & 0 & 1 & 2 \\
\hline Trombocitopenia & $\begin{array}{l}\text { Caída recuento plaquetas } \\
<30 \% \text { o nadir }<10.000 \\
\text { cél } / \mathrm{mL}\end{array}$ & $\begin{array}{l}\text { Caída recuento plaquetas } \\
30-50 \% \text { o nadir } 10.000- \\
19.000 \text { cél/mL }\end{array}$ & $\begin{array}{l}\text { Caída recuento plaquetas } \\
>50 \% \text { o nadir }>20.000 \\
\text { cél } / \mathrm{mL}\end{array}$ \\
\hline $\begin{array}{l}\text { Tiempo entre exposición } \\
\text { y trombocitopenia }\end{array}$ & $\begin{array}{l}<4 \text { días sin exposición recien- } \\
\text { te a heparina }\end{array}$ & $\begin{array}{l}\text { >10 días o tiempo no confir- } \\
\text { mado (ej sin recuento diario) }\end{array}$ & $\begin{array}{l}5 \text { a } 10 \text { días, o }<1 \text { día si expo- } \\
\text { sición reciente a heparina }\end{array}$ \\
\hline Trombosis & No & $\begin{array}{l}\text { Trombosis progresiva o recu- } \\
\text { rrente; o sospecha de nueva } \\
\text { trombosis }\end{array}$ & $\begin{array}{l}\text { Nueva trombosis confirmada; } \\
\text { o reacción anafilactoídea post } \\
\text { administración de heparina }\end{array}$ \\
\hline $\begin{array}{l}\text { Otras causas de } \\
\text { trombocitopenia }\end{array}$ & Hay otra causa confirmada & Hay otra causa posible & No hay otra causa \\
\hline
\end{tabular}

Modificado de Greinacher ${ }^{1}$. El riesgo de los paciente depende del puntaje obtenido, a saber: Riesgo bajo $(0-3$ puntos $)<1 \%$ de presentar HIT; Riesgo Intermedio (4-5 puntos): 10\% riesgo; Riesgo alto (6-8 puntos): 50\% riesgo. HIT: trombocitopenia inducida por heparina. 
alto respectivamente, independiente de la población de pacientes en quienes se aplique ${ }^{13}$. Así, se propone que sólo pacientes con riesgo moderado o alto y aquellos en quienes no se pueda aplicar el score, continúen estudio de laboratorio ${ }^{1,2}$. Además, la evaluación clínica con estimación de riesgo será relevante para tomar las decisiones terapéuticas iniciales de forma precoz en la espera de los resultados de laboratorio'.

El test diagnóstico inicial corresponde a un inmunoensayo para IgG anti-PF4/heparina. Este cuenta con una excelente sensibilidad, pero dado que no todos los pacientes con seroconversión desarrollarán trombocitopenia, su especificidad es moderada9 9 . Es por esto que algunos grupos recomiendan complementar con un examen funcional como los test de liberación de serotinina o de activación plaquetaria inducida por heparina que confirmen la presencia de anticuerpos que provocan la activación plaquetaria. Por desgracia, estos últimos están disponibles solo en centros especializados $^{9,12}$. En la Figura 1 proponemos un esquema de estudio.

En cuanto al manejo, las principales medidas son la suspensión de la heparina y el inicio de otro anticoagulante ${ }^{9,14}$. La duración de este último dependerá de la presencia de eventos trombóticos, siendo 4 semanas en los casos no complicados y 3 meses si se presenta alguna trombosis ${ }^{14}$. La guía del American College of Chest Physicians (ACCP) publicada en 2012 recomienda tres fármacos: argatroban, lepirudin y danaparoid ${ }^{9}$, ninguno disponible en Chile. Otras opciones mencionadas en las guías son la fondaparina y bivalirudina, pero en escenarios limitados. Mientras danaparoid tiene actividad predominante contra factor $\mathrm{X}$ activado, inhibiendo la formación de trombina, argatroban y lepirudina son inhibidores directos

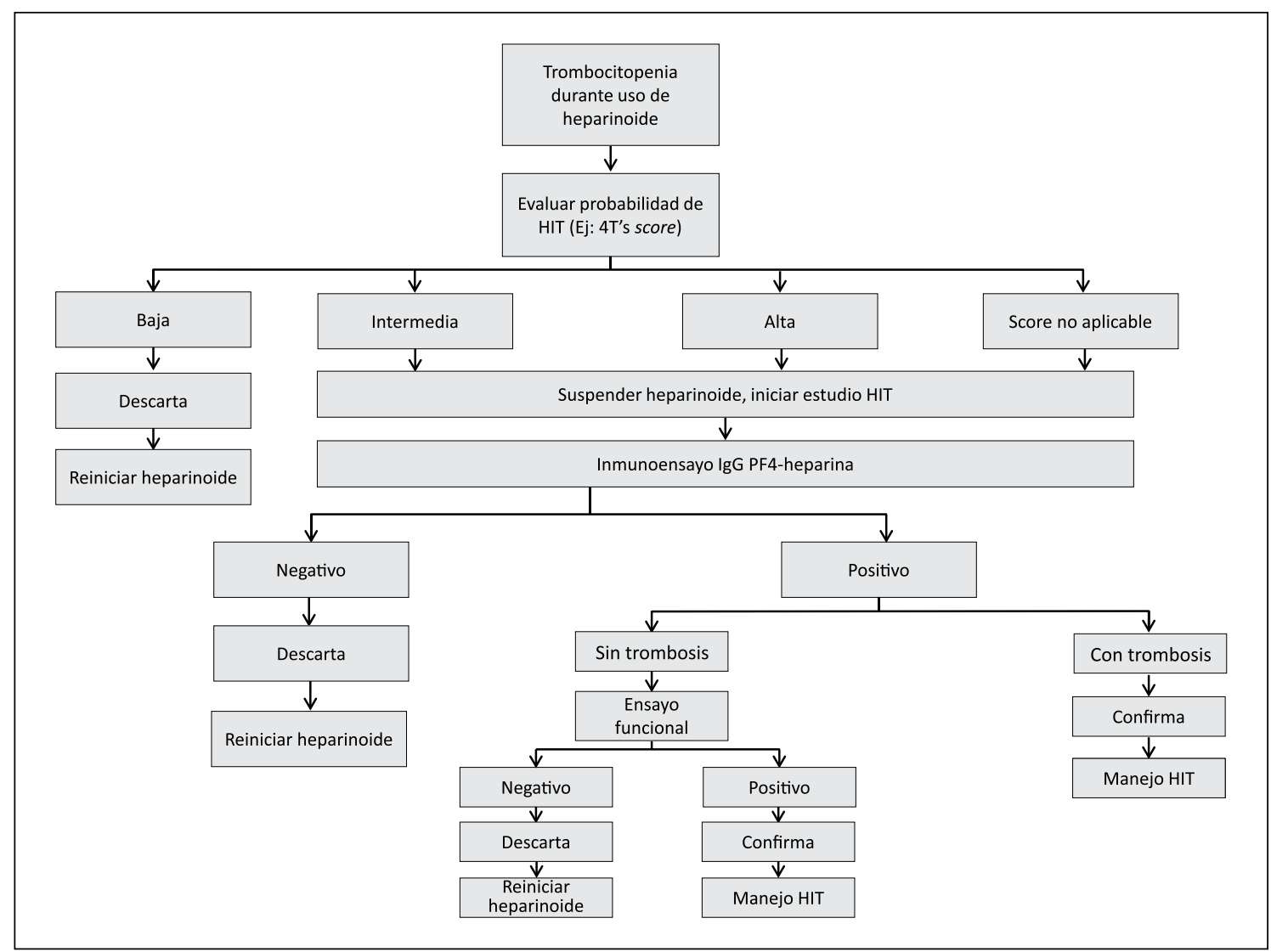

Figura 1. Algoritmo propuesto de estudio frente a paciente con sospecha de HIT en contexto de disponibilidad limitada de estudio funcional. HIT: Trombocitopenia inducida por heparina. 
de la trombina. Solo argatroban está recomendado en pacientes con insuficiencia renal. La evidencia que apoya el uso de estos anticoagulantes es limitada y corresponde a series de caso, estudios de comparación contra cohortes históricas y solo dos pequeños estudios randomizados con deficiencias metodológicas 9 .

Por otra parte, las guías recomiendan en contra del uso de antagonistas de vitamina K (AVK) ${ }^{9,14}$. Los AVK se podrían iniciar una vez alcanzado un recuento plaquetario $\geq 150.000 / \mathrm{mm}^{3}$, asociado al uso de otro anticoagulante no heparínico por al menos 5 días para evitar el efecto pro-trombótico inicial derivado de la disminución de los anticoagulantes naturales (proteína C y S) que antecede la caída de los niveles de protrombina9.

En este contexto, el uso de anticoagulantes orales de acción directa levanta interés como alternativa terapéutica ${ }^{15}$. Estudios in vitro han demostrado que tanto rivaroxaban (inhibidor del factor $\mathrm{X}$ activado) como dabigatran (inhibidor directo de trombina) no causan activación ni agregación plaquetaria en presencia de IgG anti-PF4/heparina, sugiriendo ausencia de reacción cruzada ${ }^{16,17}$. Sin embargo, la guía del ACCP no recomienda su uso por ausencia de evidencia, lo cual ha cambiado en los últimos años?.

Se han publicado series de casos de HIT manejados con rivaroxaban y dabigatran, siendo el primero el que concentra mayor número de ca$\operatorname{sos}^{18}$. En la revisión de Skelley y cols. se reportan 12 casos de HIT tratados con rivaroxaban, sin nuevos eventos trombóticos ni sangrados ${ }^{18}$.

Un estudio prospectivo multicéntrico reciente de 22 pacientes con sospecha de HIT (4Ts > 4) utilizó rivaroxaban como tratamiento. Se confirmó el diagnóstico en 12 de ellos con un ensayo funcional, completando tratamiento sólo este grupo. El esquema usado fue rivaroxaban $15 \mathrm{mg}$ 2 veces al día hasta alcanzar recuento plaquetario $\geq 150.000 / \mathrm{mm}^{3}$ y luego $20 \mathrm{mg} /$ día para completar 30 días. Durante el seguimiento, dos pacientes presentaron extensión de la trombosis ya presente al momento de iniciar rivaroxaban (uno de ellos hasta amputación) y un paciente tuvo un sangrado mayor a los 9 días. En cuanto a la trombocitopenia, el tiempo promedio de recuperación fue de 11 días. A pesar de las deficiencias metodológicas, es posible plantear que rivaroxaban podría ser efectivo en el manejo de pacientes con HIT $^{19}$.

En el caso expuesto, la paciente no presentó nuevos eventos tromboembólicos ni sangrado tras iniciar rivaroxaban $15 \mathrm{mg} \mathrm{c} / 12 \mathrm{~h}$ por 21 días y 20 $\mathrm{mg}$ /día hasta completar 3 meses, alcanzando la recuperación del recuento plaquetario 7 días tras el inicio de tratamiento.

Dado la ausencia en nuestro país de los fármacos recomendados, la alternativa de rivaroxaban (u otros anticoagulantes directos como apixaban y dabigatran) se presenta como una terapia novedosa probablemente efectiva y segura.

\section{Referencias}

1. Greinacher A. Heparin-Induced Thrombocytopenia. N Engl J Med 2015; 373 (19): 1883-4. doi: 10.1056/ NEJMc1510993. PubMed PMID: 26535525.

2. Linkins LA. Heparin induced thrombocytopenia. BMJ 2015; 350: g7566. doi: 10.1136/bmj.g7566. PubMed PMID: 25569604.

3. Scully M, Gates C, Neave L. How we manage patients with heparin induced thrombocytopenia. Br J Haematol 2016; 174 (1): 9-15. doi: 10.1111/bjh.14102. Review. PubMed PMID: 27097741.

4. Warkentin TE. Heparin-induced thrombocytopenia. Curr Opin Crit Care 2015; 21 (6): 576-85. doi: 10.1097/ MCC.0000000000000259. Review. PubMed PMID: 26539932.

5. Khandelwal S, Arepally GM. Immune pathogenesis of heparin-induced thrombocytopenia. Thromb Haemost 2016; 116 (5): 792-8. PubMed PMID: 27465274.

6. Junqueira DR, Perini E, Penholati RR, Carvalho MG. Unfractionated heparin versus low molecular weight heparin for avoiding heparin-induced thrombocytopenia in postoperative patients. Cochrane Database Syst Rev 2012; (9): CD007557. doi: 10.1002/14651858.CD007557. pub2. Review. PubMed PMID: 22972111.

7. Greinacher A, Eichler P, Lietz T, Warkentin TE. Replacement of unfractionated heparin by low-molecular-weight heparin for postorthopedic surgery antithrombotic prophylaxis lowers the overall risk of symptomatic thrombosis because of a lower frequency of heparin-induced thrombocytopenia. Blood 2005; 106 (8): 2921-2. PubMed PMID: 16204152.

8. Warkentin TE, Sheppard JA, Horsewood P, Simpson PJ, Moore JC, Kelton JG. Impact of the patient population on the risk for heparin-induced thrombocytopenia. Blood 2000; 96 (5): 1703-8. PubMed PMID: 10961867.

9. Linkins LA, Dans AL, Moores LK, Bona R, Davidson BL, Schulman S, et al; American College of Chest 
Physicians. Treatment and prevention of heparin-induced thrombocytopenia: Antithrombotic Therapy and Prevention of Thrombosis, 9th ed: American College of Chest Physicians Evidence-Based Clinical Practice Guidelines. Chest 2012; 141 (2 Suppl): e495S-530S. doi: 10.1378/chest.11-2303. Review. Erratum in: Chest. 2015 Dec;148(6):1529. PubMed PMID: 22315270; PubMed Central PMCID: PMC3278058.

10. Lubenow N, Eichler P, Lietz T, Greinacher A; Hit Investigators Group. Lepirudin in patients with heparin-induced thrombocytopenia-results of the third prospective study (HAT-3) and a combined analysis of HAT-1, HAT-2, and HAT-3. J Thromb Haemost 2005; 3 (11): 2428-36. PubMed PMID: 16241940.

11. Salter BS, Weiner MM, Trinh MA, Heller J, Evans AS, Adams DH, et al. Heparin-Induced Thrombocytopenia: A Comprehensive Clinical Review. J Am Coll Cardiol 2016; 67 (21): 2519-32. doi: 10.1016/j.jacc.2016.02.073. Review. PubMed PMID: 27230048.

12. Cuker A. Clinical and laboratory diagnosis of heparin-induced thrombocytopenia: an integrated approach. Semin Thromb Hemost 2014; 40 (1): 106-14. doi: 10.1055/s-0033-1363461. Review. PubMed PMID: 24363239.

13. Cuker A, Gimotty PA, Crowther MA, Warkentin TE. Predictive value of the 4Ts scoring system for heparin-induced thrombocytopenia: a systematic review and meta-analysis. Blood 2012; 120 (20): 4160-7. doi: 10.1182/blood-2012-07-443051. Review. PubMed PMID: 22990018; PubMed Central PMCID: PMC3501714.

14. Watson H, Davidson S, Keeling D; Haemostasis and
Thrombosis Task Force of the British Committee for Standards in Haematology. Guidelines on the diagnosis and management of heparin-induced thrombocytopenia: second edition. Br J Haematol 2012; 159 (5): 52840. doi: 10.1111/bjh.12059. Epub 2012 Oct 9. PubMed PMID: 23043677.

15. Miyares MA, Davis KA. Direct-acting oral anticoagulants as emerging treatment options for heparin-induced thrombocytopenia. Ann Pharmacother 2015; 49 (6): 735-9. doi: 10.1177/1060028015579424. Review. PubMed PMID: 25855702.

16. Krauel K, Hackbarth C, Fürll B, Greinacher A. Heparin-induced thrombocytopenia: in vitro studies on the interaction of dabigatran, Rivaroxaban, and low-sulfated heparin, with platelet factor 4 and anti-PF4/heparin antibodies. Blood 2012; 119 (5): 1248-55. doi: 10.1182/ blood-2011-05-353391. PubMed PMID: 22049520.

17. Walenga JM, Prechel M, Jeske WP, Hoppensteadt D, Maddineni J, Iqbal O, et al. Rivaroxaban -an oral, direct Factor Xa inhibitor- has potential for the management of patients with heparin-induced thrombocytopenia. Br J Haematol 2008; 143 (1): 92-9. doi: 10.1111/j.13652141.2008.07300.x. PubMed PMID: 18671707.

18. Skelley JW, Kyle JA, Roberts RA. Novel oral anticoagulants for heparin-induced thrombocytopenia. J Thromb Thrombolysis 2016; 42 (2): 172-8. doi: 10.1007/s11239016-1365-0. Review. PubMed PMID: 27102287.

19. Linkins LA, Warkentin TE, Pai M, Shivakumar S, Manji RA, Wells PS, et al. Rivaroxaban for treatment of suspected or confirmed heparin-induced thrombocytopenia study. J ThrombHaemost 2016; 14 (6): 1206-10. doi: 10.1111/jth.13330. PubMed PMID: 27061271. 